\title{
串联合成新型色烯-茚二酮类化合物
}

\author{
饶寅刘美林殷国栋* \\ (湖北师范学院化学化工学院 稀有金属化学湖北省协同创新中心 黄石 435002)
}

\begin{abstract}
摘要 报道了一种合成新型色烯-狮二酮类化合物 3 的方法. 该反应以简单易得的 2-羟基查尔酮(1)和 1,3-狮二酮(2)为 原料, 以甲苯为溶剂在无催化剂条件下进行反应. 所有化合物的结构都通过了 ${ }^{1} \mathrm{H} \mathrm{NMR},{ }^{13} \mathrm{C} N M R$, IR 和 HRMS 的表征. 该反应的可能机理为分子间迈克尔加成/分子内环化/氧化的串联过程. 目标产物 $3 \mathbf{a} \sim 3 \mathbf{h}$ 的紫外最大吸收波长在 $464 \sim$ $482 \mathrm{~nm}$ 之间.
\end{abstract}

关键词 色烯; 1,3-狮二酮; 2-着基查尔酮; 串联反应

\section{Domino Synthesis of Novel Chromene-Indanedione Derivatives}

\author{
Rao, Yin Liu, Meilin Yin, Guodong* \\ (Hubei Collaborative Innovation Center for Rare Metal Chemistry, College of Chemistry and Chemical Engineering, \\ Hubei Normal University, Huangshi 435002)
}

\begin{abstract}
A simple method for the synthesis of novel chromene-indanedione derivatives (3) has been developed by the reaction of 2-hydroxychalcones (1) with 1,3-indanedione (2) in reflux toluene under catalyst-free conditions. All the compounds were characterized by means of ${ }^{1} \mathrm{H}$ NMR, ${ }^{13} \mathrm{C}$ NMR, IR and HRMS. This reaction probably underwent a domino Michael addition/cyclization/oxidation process. The UV-Vis spectroscopy indicated that the maximum absorption wavelengths of the products $3 \mathbf{a} \sim 3 \mathbf{h}$ were at the range of $464 \sim 482 \mathrm{~nm}$.
\end{abstract}

Keywords chromene; 1,3-indanedione; 2-hydroxychalcones; domino reaction

1,3-茚二酮分子中含有 1 个活泼亚甲基和两个强吸 电子的羰基，且其中 1 个羰基容易发生烯醇异构化，正 是由于这种结构上的多样性使之被广泛地应用于有机 合成化学和药物化学领域 ${ }^{[1 \sim 4]}$. 如 Phenindione, Anisindine 和 Chlorophacinone 就是含有 1,3-狮二酮结构片段 的药物 ${ }^{[5]}$. 文献报道杯 [4]吡咯和香豆素衍生的 1,3-茚二 酮可以作为苂光分子探针来检测氰基负离子, 其主要是 利用分子间的迈克尔加成反应将碳碳双键转化为单键 来破坏分子的共轭性 ${ }^{[6]}$. 另外, 一些芳基亚甲基-1,3狮二酮也是光致变色、有机发光和有机染料等功能材料 中的结构单元 ${ }^{[9 \sim 11]}$. 然而, 在所有这些文献报道的引入 狮二酮合成方法中, 都是通过醛羰基和 1,3-狮二酮的活 泼亚甲基之间的 Knoevenagel 缩合反应得到 ${ }^{[12,13]}$. 众所 周知, 色烯 (苯并吡喃) 也是一类重要的杂环化合 物 ${ }^{[14 ~ 16]}$, 很多具有生物活性的分子和天然产物中都含
有这个结构片段 ${ }^{[17]}$. 据文献调研，色烯一狮二酮类化合 物还没有文献报道. 我们最近发现一种以 2 -羟基查尔 酩 ${ }^{[18 ~ 20]}$ 和六元环状的 1,3-二酮(或萗酚/取代苯酚)高立 体选择性地合成 2,8-二氧杂双环[3.3.1]壬烷的新方法, 该反应历经一个迈克尔加成/双环化的反应过程 ${ }^{[21,22]}$. 作为该工作的拓展，本文报道了以 2-羟基查尔酮和 1,3狮二酮为原料, 在无催化剂的条件下通过分子间迈克尔 加成/分子内环化/氧化反应串联合成新型的色烯-茚二 酮类化合物.

\section{1 结果与讨论}

首先, 我们尝试以 2 -羟基查尔酮 $(\mathbf{1 a}, 0.5 \mathrm{mmol}$ )和 1,3-狮二䣯 $(2,0.5 \mathrm{mmol})$ 在变化的条件下反应, 并发现 在甲苯中回流反应 $12 \mathrm{~h}$ 后反应原料消失, 分离得到了红 色的固体 2-(2-苯基-苯并吡喃-4-亚基)-1 $H$-狮-1,3(2H)-

\footnotetext{
*E-mail: gdyin_hbnu@163.com; gdyin@hbnu.edu.cn Received August 27, 2014; revised October 24, 2014; published online November 7, 2014.

Project supported by the Educational Commission of Hubei Province (No. D20142501) and the National Natural Science Foundation of China (No. 21102042).

湖北省教育厅重点项目基金(No. D20142501)和国家自然科学基金(No. 21102042)资助项目.
} 
二酮(3a), 产率为 $67 \%$ (表 1), 其结构通过了 ${ }^{1} \mathrm{H}$ NMR, ${ }^{13} \mathrm{C} N M R$, IR 和 HRMS 的表征. ${ }^{1} \mathrm{H}$ NMR 显示化合物 3a 在 $\delta 9.35$ 处有一个单峰, 归属为色烯环上 3-位的氢质子. 该烯氢的化学位移出现在较低场, 可能是由于存在分子 内的 $\mathrm{C}-\mathrm{H} \cdots \mathrm{O}$ 氢键相互作用, 使其化学位移值较一般 烯氢大 ${ }^{[23,24]}$. 另外, ${ }^{13} \mathrm{C} \mathrm{NMR}$ 谱显示化学位移分别在 $\delta$ 192.6 和 190.0 有两个共振信号, 归属为狮二酮中两个特 征的羰基, 且共振信号在 $\delta 192.6$ 是形成分子内氢键的 羰基. 另外发现在甲苯中降低反应温度会导致产率的明 显下降, 在其它溶剂如 $\mathrm{MeCN}, 1,2-$ 二氯乙烷(DCE), DMF, DMSO, $n$-BuOH, $t$-BuOH, $n-\mathrm{PrOH}, i-\mathrm{PrOH}, \mathrm{EtOH}$ 和 $\mathrm{MeOH}$ 中反应, 产物 3a 可以 $35 \% \sim 54 \%$ 的产率得到. 若该反应在敞开的体系中以甲苯为溶剂进行反应时，产 物 3a 的收率可以提高至 70\%.

表 1 合成化合物 $3 \mathrm{a}$ 的反应条件的笁选和优化 ${ }^{a}$

Table 1 Optimization of the reaction conditions for the synthesis of $\mathbf{3 a}$

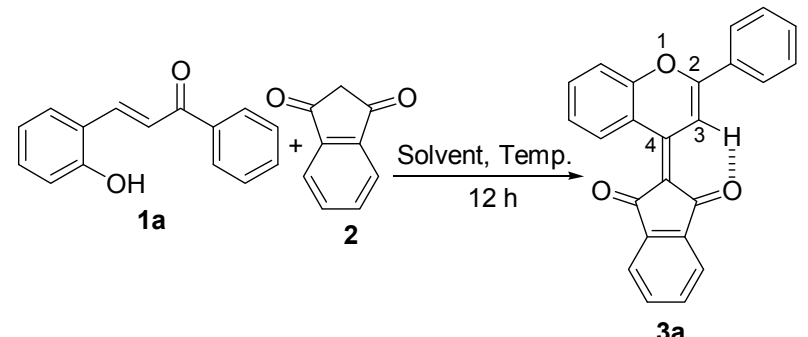

\begin{tabular}{clcc}
\hline Entry & Solvent & Temperature $/{ }^{\circ} \mathrm{C}$ & Yield $^{b} \%$ \\
\hline 1 & Toluene & Reflux & 67 \\
2 & Toluene & 80 & 36 \\
3 & MeCN & Reflux & 50 \\
4 & DCE & Reflux & 40 \\
5 & DMF & 110 & 51 \\
6 & DMSO & 110 & 50 \\
7 & $n$-BuOH & Reflux & 54 \\
8 & $t$-BuOH & Reflux & 45 \\
9 & $n$-PrOH & Reflux & 50 \\
10 & $i$-PrOH & Reflux & 38 \\
11 & EtOH & Reflux & 42 \\
12 & MeOH & Reflux & 35 \\
13 & Toluene & Reflux & $70^{c}$ \\
\hline
\end{tabular}

${ }^{a}$ All reactions were performed with 2-hydroxychalcone (1a, $\left.0.5 \mathrm{mmol}\right)$ and 1,3-indanedione $(2,0.5 \mathrm{mmol})$ in an appropriate solvent $(6 \mathrm{~mL})$ for $12 \mathrm{~h}$.

${ }^{b}$ Isolated yield. ${ }^{c}$ In an open reaction system.

为了拓展底物范围，我们以不同取代的 2-羟基查尔 酮与 1,3-狮二酮反应来考察该方法的普适性, 其中 2-羟 基查尔酮参考文献方法合成 ${ }^{[25,26]}$. 如 Eq. 1 所示, 当底物 1 中 $\mathrm{R}^{1}$ 为氢原子, $\mathrm{R}^{2}$ 为苯环上连接给电子取代基甲氧基, 反应可以很顺利地进行, 并分别以 $66 \%$ 和 $63 \%$ 产率生成 相应的产物 $3 \mathbf{b}$ 和 $3 \mathbf{c}$. 我们期望合成一些含有卤原子的 目标产物, 因为卤原子可以通过经典的偶联反应进一步
的转化为其它重要衍生物. 我们发现当苯环上连接有吸 电子的氯原子和氟原子时，相应的产物 $3 \mathrm{~d}$ 和 $3 \mathrm{e}$ 分别以 $72 \%$ 和 $67 \%$ 的产率分离得到. 萗环和杂环(呋喃环和噻 吩环)底物也适合这个反应，并以 $60 \% \sim 66 \%$ 产率生成 相应的红色固体产物 $\mathbf{3 f} \sim \mathbf{3 h}$. 若底物中 $\mathrm{R}^{2}$ 为苯环, $\mathrm{R}^{1}$ 分 别为 4-位甲氧基、5-位溴原子和 5-位氯原子，相应的产 物 3i $\sim 3 \mathbf{k}$ 也可以 $68 \% \sim 71 \%$ 的产率得到. 目标产物 $3 \mathbf{a} \sim$ 3k 中色烯环上 H-3 的化学位移的范围为 $\delta 9.10 \sim 9.47$, 两个羰基的化学位移值也如表 2 所示.

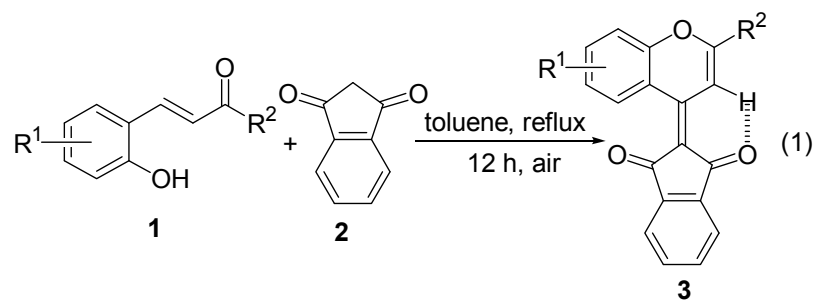

3a: $\mathrm{R}^{1}=\mathrm{H}, \mathrm{R}^{2}=\mathrm{C}_{6} \mathrm{H}_{5}, 70 \%$

3b: $\mathrm{R}^{1}=\mathrm{H}, \mathrm{R}^{2}=4-\mathrm{MeOC}_{6} \mathrm{H}_{4}, 66 \%$ 3c: $\mathrm{R}^{1}=\mathrm{H}, \mathrm{R}^{2}=3,4-(\mathrm{MeO})_{2} \mathrm{C}_{6} \mathrm{H}_{3}, 63 \%$ 3d: $\mathrm{R}^{1}=\mathrm{H}, \mathrm{R}^{2}=4-\mathrm{ClC}_{6} \mathrm{H}_{4}, 72 \%$ 3e: $\mathrm{R}^{1}=\mathrm{H}, \mathrm{R}^{2}=4-\mathrm{FC}_{6} \mathrm{H}_{4}, 67 \%$ 3f: $R^{1}=H, R^{2}=2-N a p h, 62 \%$ 3g: $R^{1}=H, R^{2}=2$-Furyl, $60 \%$ 3h: $\mathrm{R}^{1}=\mathrm{H}, \mathrm{R}^{2}=2$-Thienyl, $62 \%$ 3i: $R^{1}=4-\mathrm{OMe}, \mathrm{R}^{2}=\mathrm{C}_{6} \mathrm{H}_{5}, 68 \%$ 3j: $\mathrm{R}^{1}=5-\mathrm{Br}, \mathrm{R}^{2}=\mathrm{C}_{6} \mathrm{H}_{5}, 70 \%$ 3k: $\mathrm{R}^{1}=5-\mathrm{Cl}, \mathrm{R}^{2}=\mathrm{C}_{6} \mathrm{H}_{5}, 71 \%$

表 2 化合物 3a 3k 特征化学位移和最大吸收波长

Table 2 Characteristic chemical shift and $\lambda_{\max }$ of compounds $3 \mathbf{3 a} \sim 3 \mathbf{k}$

\begin{tabular}{ccccc}
\hline \multirow{2}{*}{ Compd. } & \multicolumn{3}{c}{ Chemical shift $(\delta)$} & \multirow{2}{*}{$\lambda_{\max } / \mathrm{nm}$} \\
\cline { 2 - 4 } & $\mathrm{H}-3$ & $\mathrm{C}=\mathrm{O}(\mathrm{H}-$ bond $)$ & $\mathrm{C}=\mathrm{O}$ & \\
\hline 3a & 9.35 & 192.6 & 190.0 & 464 \\
3b & 9.25 & 192.7 & 190.1 & 476 \\
$\mathbf{3 c}$ & 9.24 & 192.7 & 190.1 & 482 \\
$\mathbf{3 d}$ & 9.29 & 192.6 & 189.9 & 466 \\
3e & 9.33 & 192.7 & 189.9 & 464 \\
$\mathbf{3 f}$ & 9.47 & 192.7 & 190.0 & 474 \\
$\mathbf{3 g}$ & 9.10 & 192.4 & 189.9 & 474 \\
$\mathbf{3 h}$ & 9.16 & 192.5 & 189.9 & 480 \\
$\mathbf{3 i}$ & 9.23 & 192.5 & 190.0 & - \\
3j & 9.39 & 192.6 & 189.9 & - \\
3k & 9.38 & 192.6 & 189.9 & - \\
\hline
\end{tabular}

该反应的可能机理如 Scheme 1 所示. 首先, 在无催 化剂的条件下，2-羟基查尔酮(1a)和 1,3-狮二酮(2)通过 分子间的迈克尔加成反应生成相应的中间体 $\mathbf{I}^{[21,27]}$ ，再 发生分子内的环化反应生成可能的半缩醛形式中间体 $\mathbf{I I}^{[28]}$, 然后中间体 II 脱水形成色烯环 III，后者在空气氛 围中很快发生脱氢氧化成更加稳定的共轭结构 $3 \mathbf{a}$. 
<smiles>O=C(/C=C/c1ccccc1O)c1ccccc1</smiles><smiles>O=C1c2ccccc2C(=O)C1C1C=C(c2ccccc2)Oc2ccccc21</smiles><smiles>CO[C@H](O)[C@@H](O)OCc1ccccc1</smiles>

图式 1 可能的反应机理

Scheme 1 The possible reaction mechanism

我们也选择化合物 3a 3h 来研究这些红色固体的 光谱性质. 紫外可见光谱显示这些化合物在 $1.0 \times 10^{-5}$ $\mathrm{mol} / \mathrm{L}$ 的 $N, N$-二甲基甲酰胺(DMF)溶液中的最大吸收波 长在 464 482 nm. 与化合物 3a 相比, 化合物 3b, 3c 和 3f 的最大吸收波长出现 $12 \sim 18 \mathrm{~nm}$ 的红移, 这可能归因 于甲氧基和荎环的电子效应和共轭作用. 苯环上连接氯 原子和氟原子时并不明显影响该化合物的最大吸收波 长. 呋喃和噻吩环产物 $\mathbf{3 g}$ 和 $\mathbf{3 h}$ 的紫外最大吸收波长相 比较于 $3 \mathbf{a}$ 也有所增加, 其可能原因是五元杂环中未成 对的 $\mathrm{p}$-电子与碳碳双键之间存在共轭效应. 然而, 遗憾 的是这类化合物在溶液态和固态下都显示很弱的荧光 (图 1).

\section{2 结论}

本文报道了一种在无催化剂条件下有效地合成色 烯-茆二酮类化合物 3 的方法, 它是以简单易得的 2-羟 基查尔酮和 1,3-狮二酮为原料在甲苯中回流中得到. 本 文所合成的系列杂环分子均未见文献报道, 其结构通过 ${ }^{3}{ }^{1} \mathrm{H} N M R,{ }^{13} \mathrm{C}$ NMR, IR 和 HRMS 的证实. 这类化合物 的烯氢质子的化学位移在明显的低场, 表明其存在分子 内的六元环 $\mathrm{C}-\mathrm{H} \cdots \mathrm{O}$ 氢键. 该反应可能的机理为分子 间迈克尔加成/分子内环化/氧化的串联过程. 红色固体 3a $\sim 3 h$ 的紫外最大吸收波长为在 464 482 nm 之间,

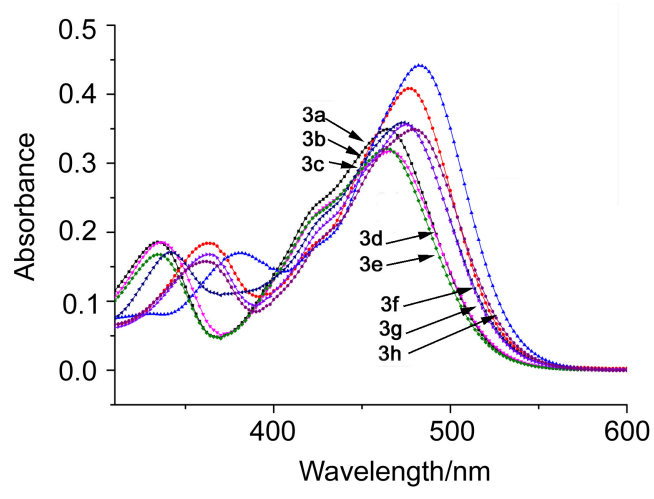

图 $13 \mathbf{3 a} \sim 3 \mathbf{h}$ 在 $\mathrm{DMF}$ 中的紫外可见光谱图 $\left(1.0 \times 10^{-5} \mathrm{~mol} / \mathrm{L}\right)$ Figure 1 UV-Vis spectroscopy for $\mathbf{3 a} \sim \mathbf{3 h}$ in DMF $\left(1.0 \times 10^{-5}\right.$ $\mathrm{mol} / \mathrm{L}$ )

在有机染料领域有潜在的应用价值.

\section{3 实验部分}

\section{1 仪器与试剂}

熔点采用泰克 X-4 型数字显示显微熔点仪测定, 温 度未经校正; ${ }^{1} \mathrm{H}$ NMR 和 ${ }^{13} \mathrm{C}$ NMR 采用 Bruker AV 300 型核磁共振仪测定, $\mathrm{CDCl}_{3}$ 为溶剂, TMS 为内标; IR 采用 Nicolet 5700 型红外分光光度仪测定( $\mathrm{KBr}$ 压片); HRMS 采用 Waters GCT Premier (ESI)或 Bruker UltrafleXtreme MALDI TOF/TOF[ 以 $\alpha$-氧基-4-羟基肉桂酸(HCCA)为基 质]型高分辨质谱仪测定; UV-Vis 采用 Hitachi U-3010 型 分光光度计测定.

所用试剂均为市售分析纯，未进一步纯化. 所用有 机溶剂在使用前均经过干燥并蒸馏提纯.

\section{2 实验方法}

目标化合物 3 的合成方法: 将 2-羟基查尔酮类化合 物(1,0.5 mmol) 和 1,3 -狮二酮 $(2,0.5 \mathrm{mmol})$ 加入装有回流 冷凝管的 $25 \mathrm{~mL}$ 烧瓶中, 再向其中加入无水甲苯 $(6 \mathrm{~mL})$, 在敞开反应体系中回流。当反应物消失后 $(12 \mathrm{~h}$, 用 TLC 监测), 将混合物冷却至室温。然后以石油醚和乙酸乙酯 为洗脱剂(体积比为 $30: 1 \sim 100: 1$ ), 通过硅胶柱柱层 析分离得到目标化合物 $\mathbf{3 a} \sim 3 \mathbf{k}$, 其波谱数据如下:

2-(2-苯基-苯并吡喃-4-亚基)-1 $H$-狮-1,3(2H)-二酮 (3a): 红色固体 $123 \mathrm{mg}$, 产率 70\%. m.p. 205 207 ${ }^{\circ} \mathrm{C}$; ${ }^{1} \mathrm{H}$ NMR $\left(300 \mathrm{MHz}, \mathrm{CDCl}_{3}\right) \delta$ : $9.35(\mathrm{~s}, 1 \mathrm{H}), 8.80(\mathrm{dd}, J=$ $1.4,8.4 \mathrm{~Hz}, 1 \mathrm{H}), 8.12 \sim 8.09(\mathrm{~m}, 2 \mathrm{H}), 7.88 \sim 7.86(\mathrm{~m}, 2 \mathrm{H})$, $7.78 \sim 7.67(\mathrm{~m}, 3 \mathrm{H}), 7.61 \sim 7.55(\mathrm{~m}, 4 \mathrm{H}), 7.51 \sim 7.45(\mathrm{~m}$, $1 \mathrm{H}) ;{ }^{13} \mathrm{C}$ NMR $\left(75 \mathrm{MHz}, \mathrm{CDCl}_{3}\right) \delta: 192.6,190.0,160.1$, $154.9,151.3,140.4,140.3,134.3,133.7,133.6,132.3$, $131.9,131.5,129.1,126.7,124.4,122.0,121.6,119.3$, 117.7, 114.7, 106.2; IR (KBr) v: 2359, 2026, 1652, 1611, 
$1501,1448,1386,1289,1101,1002,731,616 \mathrm{~cm}^{-1}$; HRMS calcd for $\mathrm{C}_{24} \mathrm{H}_{15} \mathrm{O}_{3}[\mathrm{M}+\mathrm{H}]^{+} 351.1016$, found 351.1026.

2-[2-(4-甲氧基苯基)-苯并吡喃-4-亚基]-1 H-狮1,3(2H)-二酮(3b): 红色固体 $126 \mathrm{mg}$, 产率 66\%. m.p. $227 \sim 229{ }^{\circ} \mathrm{C}$; ${ }^{1} \mathrm{H}$ NMR (300 MHz, $\left.\mathrm{CDCl}_{3}\right) \delta$ : 9.25 (s, 1H), $8.78(\mathrm{dd}, J=1.4,8.3 \mathrm{~Hz}, 1 \mathrm{H}), 8.08 \sim 8.05(\mathrm{~m}, 2 \mathrm{H}), 7.86 \sim$ $7.84(\mathrm{~m}, 2 \mathrm{H}), 7.76 \sim 7.65(\mathrm{~m}, 3 \mathrm{H}), 7.59 \sim 7.56(\mathrm{~m}, 1 \mathrm{H})$, $7.49 \sim 7.43(\mathrm{~m}, 1 \mathrm{H}), 7.07 \sim 7.03(\mathrm{~m}, 1 \mathrm{H}), 3.92(\mathrm{~s}, 3 \mathrm{H}) ;{ }^{13} \mathrm{C}$ NMR $\left(75 \mathrm{MHz}, \mathrm{CDCl}_{3}\right) \delta: 192.7,190.0,162.8,160.5$, $154.8,151.8,140.3,134.1,133.5,133.4,132.3,128.6$, $124.4,123.7,121.8,121.4,119.4,117.6,114.6,113.8$, 105.3, 55.6; IR (KBr) v: 2026, 1645, 1602, 1560, 1487, $1446,1388,1310,1256,1177,1140,1010,1014,828,732$, $607 \mathrm{~cm}^{-1}$; HRMS calcd for $\mathrm{C}_{25} \mathrm{H}_{17} \mathrm{O}_{4}[\mathrm{M}+\mathrm{H}]^{+} 381.1121$, found 381.1123 .

2-[2-(3,4-二甲氧基苯基)-苯并吡喃-4-亚基]- $1 H$-狮1,3(2H)-二酮(3c): 红色固体 $129 \mathrm{mg}$, 产率 63\%. m.p. 229 $231{ }^{\circ} \mathrm{C}$; ${ }^{1} \mathrm{H}$ NMR (300 MHz, $\left.\mathrm{CDCl}_{3}\right) \delta$ : 9.24 (s, 1H), $8.76(\mathrm{dd}, J=1.3,8.4 \mathrm{~Hz}, 1 \mathrm{H}), 7.87 \sim 7.84(\mathrm{~m}, 2 \mathrm{H}), 7.77 \sim$ $7.66(\mathrm{~m}, 4 \mathrm{H}), 7.60 \sim 7.54(\mathrm{~m}, 2 \mathrm{H}), 7.49 \sim 7.44(\mathrm{~m}, 1 \mathrm{H})$, $7.02 \sim 6.99(\mathrm{~m}, 1 \mathrm{H}), 4.05(\mathrm{~s}, 3 \mathrm{H}), 3.99(\mathrm{~s}, 3 \mathrm{H}) ;{ }^{13} \mathrm{C} \mathrm{NMR}$ $\left(75 \mathrm{MHz}, \mathrm{CDCl}_{3}\right) \delta: 192.7,190.1,160.4,154.7,152.6$, $151.6,149.3,140.3,134.1,133.5,132.3,124.4,124.0$, $121.9,121.4,120.9,119.3,117.6,113.9,111.2,108.9$, 105.6, 56.2, 56.1; IR (KBr) v: 2026, 1647, 1603, 1502, 1452, 1388, 1334, 1265, 1140, 1026, 863, 733, $612 \mathrm{~cm}^{-1}$; HRMS calcd for $\mathrm{C}_{26} \mathrm{H}_{19} \mathrm{O}_{5}[\mathrm{M}+\mathrm{H}]^{+}$411.1227, found 411.1224 .

2-[2-(4-氯苯基)-苯并吡喃-4-亚基]-1H-狮-1,3(2H)二酮(3d): 红色固体 $138 \mathrm{mg}$, 产率 $72 \%$. m.p. 215 $217{ }^{\circ} \mathrm{C} ;{ }^{1} \mathrm{H}$ NMR $\left(300 \mathrm{MHz}, \mathrm{CDCl}_{3}\right) \delta: 9.33$ (s, 1H), 8.79 $(\mathrm{dd}, J=1.4,8.3 \mathrm{~Hz}, 1 \mathrm{H}), 8.04 \sim 8.01(\mathrm{~m}, 2 \mathrm{H}), 7.88 \sim$ $7.86(\mathrm{~m}, 2 \mathrm{H}), 7.78 \sim 7.68(\mathrm{~m}, 3 \mathrm{H}), 7.59 \sim 7.45(\mathrm{~m}, 4 \mathrm{H})$; ${ }^{13} \mathrm{C}$ NMR $\left(75 \mathrm{MHz}, \mathrm{CDCl}_{3}\right) \delta: 192.7,189.9,158.7,154.8$, $150.9,140.4,140.3,138.1,134.4,133.9,133.7,132.3$, $130.0,129.4,127.8,124.6,122.1,121.7,119.2,117.6$, 115.0, 106.1; IR (KBr) v: 3076, 1655, 1605, 1499, 1304, 1093, 1002, 825, 730, $602 \mathrm{~cm}^{-1}$; HRMS calcd for $\mathrm{C}_{24} \mathrm{H}_{14} \mathrm{ClO}_{3} \quad[\mathrm{M}+\mathrm{H}]^{+}$385.0626, found 385.0620.

2-[2-(4-氟苯基)-苯并吡喃-4-亚基]-1H-狮-1,3(2H)二酮(3e): 红色固体 $123 \mathrm{mg}$, 产率 67\%. m.p. 212 $215{ }^{\circ} \mathrm{C} ;{ }^{1} \mathrm{H}$ NMR $\left(300 \mathrm{MHz}, \mathrm{CDCl}_{3}\right) \delta: 9.29$ (s, 1H), 8.79 $(\mathrm{dd}, J=1.4,8.3 \mathrm{~Hz}, 1 \mathrm{H}), 8.12 \sim 8.06(\mathrm{~m}, 2 \mathrm{H}), 7.89 \sim$ $7.84(\mathrm{~m}, 2 \mathrm{H}), 7.78 \sim 7.66(\mathrm{~m}, 3 \mathrm{H}), 7.59 \sim 7.57(\mathrm{~m}, 1 \mathrm{H})$,
$7.50 \sim 7.45(\mathrm{~m}, 1 \mathrm{H}), 7.24 \sim 7.21(\mathrm{~m}, 1 \mathrm{H}) ;{ }^{13} \mathrm{C} \operatorname{NMR}(75$ $\left.\mathrm{MHz}, \mathrm{CDCl}_{3}\right) \delta: 192.6,189.9,164.9\left(\mathrm{~d}, J_{\mathrm{C}-\mathrm{F}}=252.6 \mathrm{~Hz}\right)$, $158.9,154.7,151.0,140.3\left(\mathrm{~d}, J_{\mathrm{C}-\mathrm{F}}=2.8 \mathrm{~Hz}\right), 134.3,133.8$, $133.7,132.3,128.9\left(\mathrm{~d}, J_{\mathrm{C}-\mathrm{F}}=8.9 \mathrm{~Hz}\right), 127.8,127.7,124.5$, $122.0,121.6,119.2,117.6,116.4\left(\mathrm{~d}, J_{\mathrm{C}-\mathrm{F}}=21.9 \mathrm{~Hz}\right)$, 114.8, 105.7; IR (KBr) v: 2025, 1656, 1609, 1512, 1455 , 1286, 1224, 1102, 1003, 833, 730, $609 \mathrm{~cm}^{-1}$; HRMS calcd for $\mathrm{C}_{24} \mathrm{H}_{14} \mathrm{FO}_{3} \quad[\mathrm{M}+\mathrm{H}]^{+}$369.0921, found 369.0927.

2-[2-(2-菜基)-苯并吡喃-4-亚基]-1H-狮-1,3(2H)-二 酮(3f): 红色固体 $124 \mathrm{mg}$, 产率 $62 \%$. m.p. $221 \sim 223{ }^{\circ} \mathrm{C}$; ${ }^{1} \mathrm{H}$ NMR $\left(300 \mathrm{MHz}, \mathrm{CDCl}_{3}\right) \delta: 9.47(\mathrm{~s}, 1 \mathrm{H}), 8.80$ (dd, $J=$ $1.3,8.3 \mathrm{~Hz}, 1 \mathrm{H}), 8.62(\mathrm{~s}, 1 \mathrm{H}), 8.15 \sim 8.11(\mathrm{~m}, 1 \mathrm{H}), 8.04 \sim$ $7.97(\mathrm{~m}, 2 \mathrm{H}), 7.92 \sim 7.85(\mathrm{~m}, 3 \mathrm{H}), 7.80 \sim 7.56(\mathrm{~m}, 6 \mathrm{H})$, $7.52 \sim 7.46(\mathrm{~m}, 1 \mathrm{H}) ;{ }^{13} \mathrm{C} \mathrm{NMR}\left(75 \mathrm{MHz}, \mathrm{CDCl}_{3}\right) \delta: 192.7$, $190.0,160.1,154.9,151.3,140.4,140.3,134.8,134.3$, $133.7,133.6,133.0,132.3,129.2,129.0,128.6,128.2$, $127.8,127.4,127.1,124.5,122.8,122.0,121.6,119.3$, 117.7, 114.7, 106.6; IR (KBr) v: 3057, 1655, 1604, 1502, 1456, 1300, 1131, 1003, 861, 732, $609 \mathrm{~cm}^{-1}$; HRMS calcd for $\mathrm{C}_{28} \mathrm{H}_{17} \mathrm{O}_{3}[\mathrm{M}+\mathrm{H}]^{+}$401.1172, found 401.1176.

2-[2-(2-呋喃基)-苯并吡喃-4-亚基]- $1 H$-狮-1,3(2H)二酮(3g): 红色固体 $102 \mathrm{mg}$, 产率 60\%. m.p. 209 $211{ }^{\circ} \mathrm{C} ;{ }^{1} \mathrm{H}$ NMR $\left(300 \mathrm{MHz}, \mathrm{CDCl}_{3}\right) \delta: 9.10$ (s, 1H), 8.72 $(\mathrm{dd}, J=1.4,8.3 \mathrm{~Hz}, 1 \mathrm{H}), 7.88 \sim 7.85(\mathrm{~m}, 2 \mathrm{H}), 7.75 \sim$ $7.67(\mathrm{~m}, 4 \mathrm{H}), 7.53 \sim 7.42(\mathrm{~m}, 2 \mathrm{H}), 7.23 \sim 7.21(\mathrm{~m}, 1 \mathrm{H})$, $6.65 \sim 6.64(\mathrm{~m}, 1 \mathrm{H}) ;{ }^{13} \mathrm{C} \mathrm{NMR}\left(75 \mathrm{MHz}, \mathrm{CDCl}_{3}\right) \delta: 192.3$, $189.9,154.1,151.8,150.6,146.8,146.4,140.5,140.4$, $134.2,133.7,133.6,132.4,124.4,122.0,121.7,119.3$, $117.5,114.9,114.0,112.9,105.0$; IR (KBr) v: 1660, 1626, $1515,1461,1287,1137,1095,1005,871,753,609 \mathrm{~cm}^{-1}$; HRMS calcd for $\mathrm{C}_{22} \mathrm{H}_{13} \mathrm{O}_{4}[\mathrm{M}+\mathrm{H}]^{+} 341.0808$, found 341.0809 .

2-[2-(2-噻吩基)-苯并吡喃-4-亚基]-1H-狮-1,3(2H)二酮(3h): 红色固体 $110 \mathrm{mg}$, 产率 62\%. m.p. 205 $208{ }^{\circ} \mathrm{C}$; ${ }^{1} \mathrm{H}$ NMR $\left(300 \mathrm{MHz}, \mathrm{CDCl}_{3}\right) \delta$ : 9.16 (s, 1H), 8.74 $(\mathrm{dd}, J=1.4,8.3 \mathrm{~Hz}, 1 \mathrm{H}), 7.90 \sim 7.85(\mathrm{~m}, 3 \mathrm{H}), 7.76 \sim$ $7.62(\mathrm{~m}, 4 \mathrm{H}), 7.56 \sim 7.53(\mathrm{~m}, 1 \mathrm{H}), 7.49 \sim 7.43(\mathrm{~m}, 1 \mathrm{H})$, $7.24 \sim 7.21(\mathrm{~m}, 1 \mathrm{H}) ;{ }^{13} \mathrm{C} \mathrm{NMR}\left(75 \mathrm{MHz}, \mathrm{CDCl}_{3}\right) \delta: 192.5$, $189.9,156.0,154.4,150.9,140.4,140.3,135.5,134.2$, $133.7,133.6,132.3,131.2,129.3,128.8,124.5,122.0$, 121.6, 119.3, 117.5, 114.4, 105.5; IR (KBr) v: 1653, 1602, 1494, 1296, 1113, 1001, 865, 756, 706, $605 \mathrm{~cm}^{-1}$; HRMS calcd for $\mathrm{C}_{22} \mathrm{H}_{13} \mathrm{O}_{3} \mathrm{~S}[\mathrm{M}+\mathrm{H}]^{+}$357.0580, found 357.0574 .

2-(7-甲氧基-2-苯基-苯并吡喃-4-亚基)- $1 H$-茚1,3(2H)-二酮(3i): 红色固体 $129 \mathrm{mg}$, 产率 68\%. m.p. 
$210 \sim 211{ }^{\circ} \mathrm{C} ;{ }^{1} \mathrm{H}$ NMR $\left(300 \mathrm{MHz}, \mathrm{CDCl}_{3}\right) \delta: 9.23(\mathrm{~s}, 1 \mathrm{H})$, $8.75(\mathrm{~d}, J=9.2 \mathrm{~Hz}, 1 \mathrm{H}), 8.09 \sim 8.06(\mathrm{~m}, 2 \mathrm{H}), 7.85 \sim 7.82$ (m, $2 \mathrm{H}), 7.68 \sim 7.65(\mathrm{~m}, 2 \mathrm{H}), 7.55 \sim 7.53(\mathrm{~m}, 3 \mathrm{H}), 7.08 \sim$ $7.01(\mathrm{~m}, 2 \mathrm{H}), 3.98(\mathrm{~s}, 3 \mathrm{H}) ;{ }^{13} \mathrm{C} \mathrm{NMR}\left(75 \mathrm{MHz}, \mathrm{CDCl}_{3}\right) \delta$ : $192.5,190.0,164.6,159.5,156.9,151.1,140.2,133.7$, $133.3,133.2,131.6,131.3,128.9,126.4,121.7,121.3$, 113.7, 113.2, 113.1, 105.8, 100.0, 55.9; IR (KBr) v: 2356, 1657, 1620, 1498, 1447, 1345, 1305, 1236, 1031, 824, 612 $\mathrm{cm}^{-1}$; HRMS calcd for $\mathrm{C}_{25} \mathrm{H}_{17} \mathrm{O}_{4}[\mathrm{M}+\mathrm{H}]^{+}$381.1121, found 381.1121 .

2-(6-溴-2-苯基-苯并吡喃-4-亚基)- $1 H$-狮-1,3(2H)二酮 $(\mathbf{3 j})$ : 红色固体 $150 \mathrm{mg}$, 产率 70\%. m.p. 280 $282{ }^{\circ} \mathrm{C} ;{ }^{1} \mathrm{H}$ NMR $\left(300 \mathrm{MHz}, \mathrm{CDCl}_{3}\right) \delta: 9.39(\mathrm{~s}, 1 \mathrm{H}), 9.13$ $(\mathrm{d}, \quad J=2.3 \mathrm{~Hz}, 1 \mathrm{H}), 8.08 \sim 8.05(\mathrm{~m}, 2 \mathrm{H}), 7.93 \sim 7.80(\mathrm{~m}$, $3 \mathrm{H}), 7.72 \sim 7.69(\mathrm{~m}, 2 \mathrm{H}), 7.56 \sim 7.54(\mathrm{~m}, 3 \mathrm{H}), 7.49 \sim 7.46$ $(\mathrm{m}, 1 \mathrm{H}) ;{ }^{13} \mathrm{C}$ NMR $\left(75 \mathrm{MHz}, \mathrm{CDCl}_{3}\right) \delta: 192.6,189.9$, $159.9,153.7,149.4,140.4,136.9,134.6,134.0,133.9$, $132.0,131.3,129.2,126.6,122.4,121.8,120.8,119.3$, 117.4, 115.4, 105.9; IR (KBr) v: 3071, 1657, 1609, 1509, $1450,1353,1293,1227,1011,732 \mathrm{~cm}^{-1}$; HRMS calcd for $\mathrm{C}_{24} \mathrm{H}_{14} \mathrm{BrO}_{3}[\mathrm{M}+\mathrm{H}]^{+}$429.0121, found 429.0111.

2-(6-氯-2-苯基-苯并吡喃-4-亚基)- $1 H$-狮-1,3(2H)二酮 $(3 \mathbf{k})$ : 红色固体 $137 \mathrm{mg}$, 产率 71\%. m.p. 281 $283{ }^{\circ} \mathrm{C} ;{ }^{1} \mathrm{H}$ NMR $\left(300 \mathrm{MHz}, \mathrm{CDCl}_{3}\right) \delta: 9.38(\mathrm{~s}, 1 \mathrm{H}), 8.97$ (d, $J=2.4 \mathrm{~Hz}, 1 \mathrm{H}), 8.08 \sim 8.05(\mathrm{~m}, 2 \mathrm{H}), 7.92 \sim 7.84(\mathrm{~m}$, $2 \mathrm{H}), 7.73 \sim 7.66(\mathrm{~m}, 3 \mathrm{H}), 7.56 \sim 7.51(\mathrm{~m}, 4 \mathrm{H}) ;{ }^{13} \mathrm{C} \mathrm{NMR}$ $\left(75 \mathrm{MHz}, \mathrm{CDCl}_{3}\right) \delta: 192.6,189.9,160.0,153.3,149.6$, $140.4,134.1,134.0,133.9,132.0,131.5,131.3,129.9$, 129.2, 126.6, 122.4, 121.8, 120.4, 119.1, 115.3, 105.8; IR (KBr) $v$ : 3068, 1656, 1607, 1509, 1448, 1350, 1291, 1008, $722 \mathrm{~cm}^{-1}$; HRMS calcd for $\mathrm{C}_{24} \mathrm{H}_{14} \mathrm{ClO}_{3}[\mathrm{M}+\mathrm{H}]^{+}$ 385.0626, found 385.0623 .

辅助材料(Supporting Information) 化合物 $\mathbf{3 a} \sim 3 \mathbf{k}$ 的 核磁共振氢谱和核磁共振碳谱图. 这些材料可以免费从 本刊网站(http://sioc-journal.cn/)上下载.

\section{References}

[1] Lee, C.-J.; Sheu, C.-N.; Tsai, C.-C.; Wu, Z.-Z.; Lin, W. Chem. Commun. 2014, 50, 5304.

[2] Hansen, D. B.; Joullie, M. M. Chem. Soc. Rev. 2005, 34, 408.

[3] Shirini, F.; Beigbaghlou, S. S.; Atghia, S. V.; Mousazadeh, S. A. Dyes Pigm. 2013, 97, 19.

[4] Yang, S. X.; Chen, J.; Wu, X. Y.; Deng, H. M.; Shao, M.; Zhang,
H.; Cao, W. G. Chin. J. Org. Chem. 2010, 30, 1521.

(杨树新, 陈杰, 吴小余, 邓红梅, 邵敏, 张慧, 曹卫国, 有机化 学, 2010, 30, 1521.)

[5] Naisbitt, D. J.; Farrell, J.; Chamberlain, P. J.; Hopkins, J. E.; Berry, N. G.; Pirmohamed, M.; Park, B. K. J. Pharmacol. Exp. Ther. 2005, 313, 1058.

[6] Kim, S.-H.; Hong, S.-J.; Yoo, J.; Kim, S. K.; Sessler, J. L.; Lee, C.-H. Org. Lett. 2009, 11, 3626.

[7] Nishiyabu, R.; Anzenbacher, P. Org. Lett. 2006, 8, 359.

[8] Zhou, X.; Lv, X.; Hao, J.; Liu, D.; Guo, W. Dyes Pigm. 2012, 95, 168.

[9] Tehfe, M.-A.; Dumur, F.; Graff, B.; Gigmes, D.; Fouassier, J.-P.; Lalevée, J. Macromolecules 2013, 46, 3332.

[10] Kulinicha, A. V.; Derevyankoa, N. A.; Mikitenkoa, E. K.; Ishchenko, A. A. J. Phys. Org. Chem. 2011, 24, 732.

[11] Duan, Y. J.; Liu, J. L.; Wang, C. L. Chin. J. Org. Chem. 2010, 30, 988 (in Chinese). (段义杰, 刘建利, 王翠玲, 有机化学, 2010, 30, 988.)

[12] Seniutinas, G.; Tomašiūnas, R.; Czaplicki, R.; Sahraoui, B.; Daškevičienė, M.; Getautis, V.; Balevičius, Z. Dyes Pigm. 2012, 95, 33.

[13] Tong, J.; Wang, Y.; Mei, J.; Wang, J.; Qin, A.; Sun, J. Z.; Tang, B. Z. Chem. Eur. J. 2014, 20, 4661.

[14] Shi, D. Q.; Wang, X. S.; Tu, S. J. Chin. J. Org. Chem. 2002, 22, 1053 (in Chinese). (史达清, 王香善, 屠树江, 有机化学, 2002, 22, 1053.)

[15] Jiang, S. L.; Han, L. Chin. J. Org. Chem. 2012, 32, 1129 (in Chinese). (蒋绍亮, 韩亮, 有机化学, 2012, 32, 1129.)

[16] Luan, C. J.; Wang, J. Q.; Zhang, G. H.; Wang, W.; Tang, S. G.; Guo, C. Chin. J. Org. Chem. 2011, 31, 860 (in Chinese). (栾长军, 王建强, 张国华, 王伟, 唐拾贵, 郭成, 有机化学, 2011, 31, 860.)

[17] Khoshneviszadeh, M.; Edraki, N.; Miri, R.; Foroumadi, A.; Hemmateenejad, B. Chem. Biol. Drug Des. 2012, 79, 442.

[18] Yin, G. D.; Lai, T. T.; Yan, Z. S.; Chen, H.; Zheng, J.; Tao, Q. Tetrahedron 2013, 69, 2430.

[19] Yin, G. D.; Shi, H. Q.; Xu, L. Q. Y.; Wei, X. H.; Tao, Q. Synthesis 2013, 45, 334.

[20] Rao, Y.; Li, Z. X.; Yin, G. D. Green Chem. 2014, 16, 2213.

[21] Yin, G. D.; Ren, T. B.; Rao, Y.; Zhou, Y. F.; Li, Z. X.; Shu, W. M.; Wu, A. X. J. Org. Chem. 2013, 78, 3132.

[22] Rao, Y.; Yin, G. D. Org. Biomol. Chem. 2013, 11, 6029.

[23] Sashidhara, K. V.; Kumar, A.; Agarwal, S.; Kumar, M.; Kumar, B.; Sridharc, B. Adv. Synth. Catal. 2012, 354, 1129.

[24] Głowacki, E. D.; Voss, G.; Demirak, K.; Havlicek, M.; Sünger, N.; Okur, A. C.; Monkowius, U.; Gąsiorowski, J.; Leonat, L.; Sariciftci, N. S. Chem. Commun. 2013, 49, 6063.

[25] Yin, G. D.; Fan, L.; Ren, T. B.; Zheng, C. Y.; Tao, Q.; Wu, A. X.; She, N. F. Org. Biomol. Chem. 2012, 10, 8877.

[26] Mazimba, O.; Masesane, I. B.; Majinda, R. R. Tetrahedron Lett. 2011, 52, 6716.

[27] Ray, S. K.; Singh, P. K.; Molleti, N.; Singh, V. K. J. Org. Chem. 2012, 77, 8802 .

[28] Liu, Y. B.; Liu, X. H.; Wang, M.; He, P.; Lin, L. L.; Feng, X. M. J. Org. Chem. 2012, 77, 4136. 